\title{
Sedation protocols in the pediatric intensive care unit: fact or fiction?
}

\author{
Corrine R. Balit ${ }^{1}$, Jessica M. LaRosa ${ }^{2}$, Jacqueline S. M. Ong ${ }^{3,4}$, Sapna R. Kudchadkar ${ }^{2,5,6}$ \\ ${ }^{1}$ Paediatric Intensive Care Unit, John Hunter Hospital, Newcastle, Australia; ${ }^{2}$ Department of Anesthesiology and Critical Care Medicine, Johns \\ Hopkins University School of Medicine, Baltimore, MD, USA; ${ }^{3}$ Khoo Teck Puat-National University Children's Medical Institute, National \\ University Hospital, Singapore, Singapore; ${ }^{4}$ Department of Paediatrics, Yong Loo Lin School of Medicine, National University of Singapore, \\ Singapore, Singapore; ${ }^{5}$ Department of Pediatrics, Johns Hopkins University School of Medicine, Baltimore, MD, USA; ${ }^{6}$ Department of Physical \\ Medicine \& Rehabilitation, Johns Hopkins University School of Medicine, Baltimore, MD, USA \\ Contributions: (I) Conception and design: CR Balit; (II) Administrative support: C Levine; (III) Provision of study materials or patients: None; (IV) \\ Collection and assembly of data: CR Balit, JM LaRosa, JSM Ong; (V) Data analysis and interpretation: All authors; (VI) Manuscript writing: All \\ authors; (VII) Final approval of manuscript: All authors. \\ Correspondence to: Dr. Corrine R. Balit, B. Pharm, MBBS (Hons), FCICM. Director, Paediatric Intensive Care Services, John Hunter Hospital, \\ Locked Bag 1, HRMC, Newcastle, NSW 2310, Australia. Email: Corrine.Balit@health.nsw.gov.au.
}

\begin{abstract}
Comfort of the critically unwell pediatric patient is paramount to ensuring good outcomes. Analgesia-based, multimodal sedative approaches are the foundation for comfort, whereby pain is addressed first and then sedation titrated to a predefined target based on the goals of care. Given the heterogeneity of patients within the pediatric critical care population, the approach must be individualized based on the age and developmental stage of the child, physiologic status, and degree of invasive treatment required. In both the adult and pediatric intensive care unit (PICU), sedation titration is practiced as standard of care to meet therapeutic goals with a focus on facilitating early rehabilitation and extubation while avoiding underand over-sedation. Sedation protocols have been developed as methods to reduce variability and optimize goal-directed therapy. Components of a sedation protocol include routine analgesia and sedation scoring with validated tools at specified intervals and a predefined algorithm that allows the titration of analgesia and sedation based on those assessments. Sedation protocols are designed to improve communication and documentation of sedation goals while also empowering the bedside team to respond rapidly to changes in a patient's clinical status. Previously it was thought that sedation protocols would consistently reduce duration of mechanical ventilation (MV) and length of stay (LOS) for patients in the PICU, however, this has not been the case. Nonetheless, introduction of sedation protocols has provided several benefits, including: (I) reduction in benzodiazepine usage; (II) improvements in interprofessional communication surrounding sedation goals and management of sedation goals; and (III) reductions in iatrogenic withdrawal symptoms. Successful implementation of sedation protocols requires passionate clinical champions and a robust implementation, education, and sustainability plan. Emerging evidence suggests that sedation protocols as part of a bundle of quality improvement initiatives will form the basis of future studies to improve short- and long-term outcomes after PICU discharge. In this review, we aim to define sedation protocols in the context of pediatric critical care and highlight important considerations for clinical practice and research.
\end{abstract}

Keywords: Clinical protocol; pediatric intensive care unit (PICU); sedatives

Submitted Oct 10, 2020. Accepted for publication Mar 03, 2021.

doi: $10.21037 /$ tp-20-328

View this article at: http://dx.doi.org/10.21037/tp-20-328 


\section{Introduction}

Optimizing the comfort of pediatric patients during a critical illness is an essential facet of day-to-day care in the pediatric intensive care unit (PICU). Children requiring PICU care are often subject to painful, invasive interventions that are life-sustaining. Thus, maintaining physical and psychological comfort for not only the child, but also their family and the clinical care team, is of paramount importance. Apart from non-pharmacologic options, the delivery of sedation and analgesia in this environment is considered to be the standard of care (1).

Optimum delivery of comfort in the complex ecosystem of the PICU remains elusive. A great deal has been published on (I) standardizing measures of comfort; (II) the best nonpharmacologic and pharmacologic therapies to use; (III) the best way to deliver these therapies; and (IV) how to ensure quick and appropriate responses to changes in a patient's comfort level. However, immense variability exists and key questions remain unanswered. Should the delivery of sedation and analgesia be at an individual clinician's discretion, or should a predefined protocol be followed that reduces subjectivity? Should determining comfort levels in the intensive care unit (ICU) be physician-, nurse-, or parent-led?

The use of defined sedation protocols has been proposed as one way to reduce variability in analgesia and sedation for critically ill patients. For the purposes of this review, we use the term sedation protocol to delineate a written, approved algorithm that is consistently utilized for patients who require continuous sedation and/or analgesia. Ideally, a standardized process could improve patient comfort, reduce subjective assessments, and improve outcomes during admission and post-ICU discharge. Despite this reasonable aim, significant controversy remains around the efficacy of such protocols. To understand why, we need to closely examine the challenges of measuring comfort, analgesia, and sedation delivery and what outcome measures are important to determine efficacy. The aim of this review will be to evaluate the potential benefit of sedation protocols and to identify targets for intervention and research.

\section{Goals and importance of analgesia and sedation in the PICU}

The aim of analgesia and sedation in the PICU is twofold: (I) to treat pain and (II) to ensure patient safety and comfort during invasive treatments (1). Other possible goals include optimizing patient-ventilator synchrony, reducing oxygen demand, line and tube maintenance, and reducing anxiety $(2,3)$. Although every patient requires adequate analgesia for noxious stimuli (e.g., the endotracheal tube), sedative needs may vary widely from patient to patient depending on the goals of care.

As such, the pendulum for ideal depth of sedation and analgesia has swung back and forth over time. Initially, it was widely held that young children and infants did not experience the same degree of pain or discomfort as adults. As a result, pediatric patients often received minimal sedation and analgesia. Poorer outcomes were then noted with undertreated pain and anxiety, including delayed healing and increased stress on patients and caregivers (4). With these findings, children were subsequently more deeply sedated with a variety of agents, most commonly benzodiazepines and opioids (5). Increased sedation resulted in a new set of problems with clinical impact, including prolonged mechanical ventilation (MV) (6), increased iatrogenic withdrawal syndrome (IWS) (7,8), delirium (9), and the potential negative ramifications of these medications on immature and developing brains $(8,10)$. Given the increasingly deleterious effects of over-sedation, the most recent shift in sedation goals has been to titrate sedation to minimum but effective targets, with an emphasis on early extubation and rehabilitation while maintaining optimal analgesia.

Consensus guidelines from the United Kingdom (3) and the European Society of Pediatric and Neonatal Intensive Care (ESPNIC) (11) have all advocated similar recommendations. Firstly, pain should be assessed with age-appropriate validated pain scales to provide consistency between clinicians and allow for evaluation of an intervention's effect. Commonly used pain assessments in the PICU (10) include: (I) COMFORT Score, (II) Faces, Legs, Activity, Cry and Consolability (FLACC) score, and (III) Multidimensional Assessment of Pain Scale. Pain assessments should be performed regularly, particularly if the child is on an analgesic infusion, and in conjunction with patient and family assessment (11). Thus, a key component of sedation management in the PICU is that pain should be treated before targeting sedation depth, i.e., analgesia-based sedation. Clinical cues for pain or agitation from children have significant overlap, but attempting to distinguish between them allows clearer setting of goals and choice of therapies.

Secondly, an optimal sedation goal should be determined for the patient by carefully considering clinical status and 
Table 1 Comparison of commonly used sedation assessment tools in the PICU

\begin{tabular}{|c|c|c|c|c|}
\hline Dimension & SBS & COMFORT & COMFORT-B & RASS \\
\hline Features & $\begin{array}{l}\text { Response to voice, } \\
\text { gentle touch, or } \\
\text { noxious stimuli }\end{array}$ & $\begin{array}{l}\text { Domains are scored on a Likert scale from } \\
1 \text { to } 5 \text {. Includes: alertness, calmness, } \\
\text { respiratory response, physical movement, } \\
\text { muscle tone, and facial tension. Also } \\
\text { includes physiologic variables of MAP and } \\
\text { heart rate }\end{array}$ & $\begin{array}{l}\text { Same domains as Comfort } \\
\text { score except without } \\
\text { physiologic variables (MAP } \\
\text { and heart rate) }\end{array}$ & $\begin{array}{l}\text { Response to voice } \\
\text { and touch }\end{array}$ \\
\hline Score range & $\begin{array}{l}\text { Numeric rating: }-3 \text { to } \\
+2\end{array}$ & $\begin{array}{l}\text { Numeric rating. Each domain is scored out } \\
\text { of } 5 \text {. Range is } 8 \text { to } 40\end{array}$ & $\begin{array}{l}\text { Numeric rating. Each domain } \\
\text { is scored out of } 5 \text {. Range is } 6 \\
\text { to } 30\end{array}$ & $\begin{array}{l}\text { Numeric rating: } 4 \text { to } \\
-4\end{array}$ \\
\hline $\begin{array}{l}\text { Score } \\
\text { interpretation }\end{array}$ & $\begin{array}{l}\text { More negative scores } \\
=\text { sedated state; } \\
\text { more positive scores } \\
=\text { agitated state; } \\
\text { score of } 0=\text { awake } \\
\text { and calm }\end{array}$ & $\begin{array}{l}8 \text { to } 17: \text { over-sedated; } 17 \text { to } 26: \text { adequately } \\
\text { sedated; } 27 \text { to } 40: \text { under-sedated }\end{array}$ & $\begin{array}{l}6 \text { to } 10: \text { over-sedated; } 11 \text { to } \\
\text { 23: moderately sedated; } 24 \\
\text { to } 30 \text { : little sedation }\end{array}$ & $\begin{array}{l}\leq-3 \text { : over-sedated; } \\
-2 \text { to } 0: \text { adequately } \\
\text { sedated }\end{array}$ \\
\hline
\end{tabular}

PICU, pediatric intensive care unit; SBS, State Behavioral Scale; RASS, Richmond Agitation and Sedation Score; MAP, mean arterial blood pressure.

disease severity and using validated sedation scoring tools $(3,11)$. Achieving a targeted sedation level can often be challenging as it depends on the child's neurodevelopmental stage, illness severity, and by association the degree of invasive support that is required. For example, some patients may require complete immobility with deep sedation for physiologic stability, whereas others may tolerate MV and endotracheal intubation with only opioid therapy. Once the desired level of sedation has been established, it should be assessed regularly with validated scales (3). Sedative doses should then be titrated based on the assessed depth of sedation and adjusted accordingly.

\section{Sedation assessment tools}

A central component of all sedation protocols is the ability to measure a patient's sedation level at regular intervals using a reproducible, systematic, and validated approach. Utilization of standardized assessment tools enables consistent communication between PICU team members and prevention of over- or under-sedation $(1,11)$.
However, there is no gold standard tool for achieving target sedation goals (12). The challenge of such a tool in pediatrics is that it must be validated for a wide range of ages and developmental stages. Additionally, such tools need to fit within local work practices and be reproducible between various clinicians. The interval for regular assessments is defined by the scale being used and the protocol in place, but general recommendations are that it be completed every 4 to 8 hours or as indicated by the child's clinical condition (11).

Currently, the three validated pediatric sedation assessment tools for children supported on MV are: (I) the State Behavioral Assessment Scale (SBS); (II) the COMFORT Scale and its modification, the COMFORT-B Scale; and (III) the Richmond Agitation Sedation Scale (RASS) (Table 1). The SBS provides a numeric rating for a standardized range of defined behaviors in response to voice, gentle touch, or noxious stimuli (13). The scale ranges from agitated $(+2)$ to unresponsive $(-3)(13)$. The COMFORT Scale (14) was created to evaluate distress in eight domains: alertness, calmness/agitation, respiratory 
response, physical movement, mean arterial blood pressure (MAP), heart rate, muscle tone, and facial tension. These domains are evaluated on a Likert scale from 1 to 5 , from which patients receive a cumulative score (14). Given concerns that physiologic variables are influenced by medications administered in the PICU, the COMFORT-B Scale was developed and validated to eliminate physiologic variables from the score (15). The RASS is a responsiveness scale that assesses sedation and agitation and was recently validated for use in critically ill children. It is a single value numeric score that ranges from -5 to 4 to describe a patient's behavior from unarousable to combative in response to voice and touch. The RASS is unique in that it is the only sedation score that incorporates eye contact in response to verbal stimuli (16). No studies have directly compared these three validated sedation assessment tools.

\section{Components and goals of a sedation protocol}

Sedation protocols are used to achieve goal-directed, patient-specific sedation and analgesia for each clinical scenario while minimizing both under- and over-sedation. Frequently, physicians use nonstandardized practices to titrate sedation in critically ill children, leading to significant practice variation in terms of drug choice, method of drug delivery, and adherence to protocols (5). Clinicians and researchers anticipated that a formulated algorithm incorporating validated scores for sedation and pain and the use of regular assessments to guide medication titration would provide substantial benefit. Two types of standardized sedation strategies have been studied in critically ill children: protocol-directed sedation and daily sedation interruption. Protocol-directed sedation is ordered by a physician with specific goals for a patient's sedation level and is implemented by a member of the healthcare team, often bedside nursing. The patient's sedation level is assessed on a regular time interval with a validated sedation assessment tool, and sedative agents are titrated to achieve the targeted sedation goal (17). Such protocols allow the interprofessional team to set goals and transfer decisionmaking authority for analgesia and sedation to bedside clinicians, usually nurses (18). Daily sedation interruption requires a period of time each day that a patient's sedation is paused or decreased to achieve a goal level of alertness defined by a sedation assessment tool (19). The aims of sedation interruption are to reduce the total amount of sedation exposure and facilitate early weaning from the ventilator.
The reported benefits of a sedation protocol are wideranging. Sedation protocols introduce standardization of assessments of pain and sedation (10), empower bedside clinicians to respond to those assessments, and allow for a common language to be spoken regarding analgesia and sedation within the interdisciplinary team $(18,20)$. These protocols give priority to the issue of a child's comfort. Other reported benefits are more ambitious, including improvements in duration of $M V$ and reduced ICU length of stay (LOS) (21). Although many benefits of sedation protocols have been reported, they have not always been supported by strong evidence. The reported benefits are mostly extrapolated from adult studies and are listed in Box 1.

Box 1 Reported benefits from implementation of a sedation protocol

Decreased duration of MV
Decreased ICU LOS
Decreased hospital LOS
Decreased incidence and severity of delirium
Earlier mobilization
Reduced over- and under-sedation
Decreased sedative and opioid doses
Decreased incidence of iatrogenic drug withdrawal
Improved communication of sedation goals in the interdisciplinary
team
Improved documentation of sedation goals

MV, mechanical ventilation; ICU, intensive care unit; LOS, length of stay.

\section{What is the evidence for a sedation protocol?}

In an international survey of PICUs, sedation scoring tools were used in $70 \%$ of PICUs, but only $42 \%$ reported using them as part of routine daily rounds and patient care goals (5). Only $27 \%$ of PICUs had written sedation protocols with treatment algorithms in place (5). In the adult ICU literature, the results of protocol-directed sedation studies are conflicting. Individual adult studies demonstrate reductions in duration of MV, ICU LOS, and mortality (22-24); however, a large systematic review and meta-analysis of protocol-directed sedation did not 
reproduce the same benefit (17).

The largest study in the pediatric population to assess protocol-directed sedation is the Randomized Evaluation of Sedation Titration for Respiratory Failure (RESTORE) trial (25). RESTORE was an unblinded, multicenter, cluster-randomized, prospective clinical trial conducted between 2009 and 2013 that enrolled 2,449 pediatric patients receiving MV from 31 US PICUs. PICUs were randomized into either the study group or a control group. The study group included a nurse-titrated sedation protocol that incorporated prescriber-directed SBS, regular arousal assessments utilizing SBS, and daily extubation readiness tests. PICUs in the control group continued to manage sedation in their usual nonstandardized way. The primary outcome, duration of $\mathrm{MV}$, did not differ between the groups. There were also no differences in PICU LOS, hospital LOS, in-hospital mortality, inadequate sedation management, or clinically significant IWS. In a secondary exploratory analysis, patients in the intervention group were exposed to fewer classes of sedative agents ( 2 vs. 3 agents) and fewer cumulative days of opioid administration (9 vs. 10 days). The percentage of study days in which patients were awake and calm while intubated was higher in the intervention group ( $86 \%$ vs. $75 \%$ ), though the intervention group had a greater percentage of days with a higher pain score (50\% vs. $23 \%$ ) and higher SBS $(60 \%$ vs. $40 \%$ ). Additional findings included an increase in postextubation stridor and immobility-related pressure ulcers in the control group of patients. Reducing these potential sedation-related events could be considered a potential benefit of protocolized sedation. Ultimately, the authors concluded that a nurse-implemented, goal-directed sedation protocol did not reduce the duration of MV from that with usual care (25), but that children can be more awake and calm without harm. In a subsequent followup study of 1,360 patients, the authors evaluated postdischarge outcomes of children who were randomized to a sedation protocol or usual care. There were no difference in functional status, quality of life, or indication of posttraumatic stress disorder between the groups. The authors concluded that a sedation strategy in which patients are more awake does not produce long-term harm (26).

Much of the remaining evidence regarding sedation protocols in pediatrics has come from before-and-after protocol implementation studies with different outcome measures (18,20,21,27-33) (Table 2). Similar to the adult literature, these studies have produced mixed results with no clear benefit. Prior to the RESTORE study, Poh and co-authors (34) completed a systematic review of sedation protocols in pediatric critical care. The driver for the review was that many societies had included sedation protocols in their standard recommendations to improve sedation practice and standardize care with poor supportive evidence. Within the studies reviewed by Poh et al. (34), some reported benefits included decreasing unplanned extubation, IWS, and sedation doses and duration. There were no consistent positive results in terms of mortality, duration of MV, or LOS. Most studies were single center, and hence applicability to other PICUs was difficult. Another challenge was the lack of a clear definition for sedation-related adverse events.

No evidence in the pediatric literature has shown that the use of a protocol reduces duration of $\mathrm{MV}$ or PICU LOS. Most studies, including the large RESTORE study (25), have shown no difference in these outcome measures (25,27-29). The exceptions were one Korean study, which showed a decrease in duration of MV (21), and an Australian study, which demonstrated an increase in duration of MV (31). In the latter study, the authors reported a significant number of younger children in the post-implementation phase who were less likely to be extubated early post-cardiac surgery. That group potentially influenced their result. Aitken et al. (17) examined randomized controlled trials (RCT) of protocoldirected sedation in both adults and children, including three adult studies and the pediatric RESTORE study. All of these studies compared protocol-directed sedation with usual care and found no evidence of difference in MV duration and no clear evidence of difference in ICU mortality, hospital mortality, ICU LOS, or unplanned extubations.

\section{Are we asking the right questions about sedation protocols?}

The pediatric literature on sedation protocols leads us to ask: are MV duration and PICU LOS the right outcomes to assess when examining the benefit of minimal but effective sedation? Both of these variables are discrete time measurements that are affected by many factors not captured in the studies and that may be difficult to measure. For example, ventilation practice, hospital throughput, staffing ratios, and clinician preferences can all influence these outcome measures and hence make it difficult to show a benefit related to changes in sedation. In addition, all of the studies compared the use of a sedation protocol to 


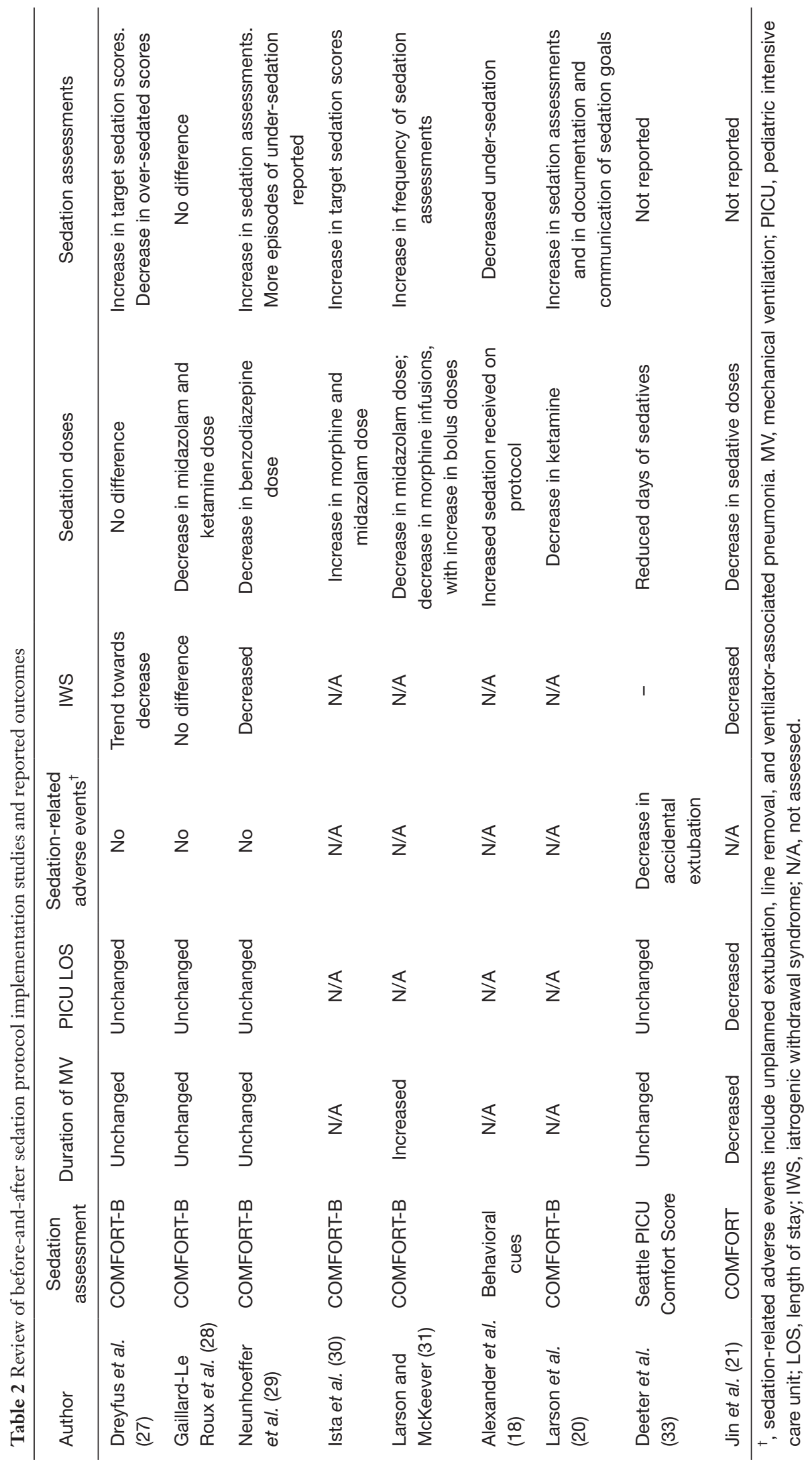


"usual" or "standard" care. However, standard care has no consistent definition. An important consideration regarding standard care is that mortality in pediatric critical care is very low (35) and outcomes are improving. It is possible that "standard care" in pediatric critical care has advanced to a point that a meaningful benefit cannot be shown in the outcomes measured.

Nonetheless, some benefits of sedation protocols have been shown. One positive outcome that was demonstrated in several studies was the reduction in use of benzodiazepines, midazolam in particular, with no increase in harm to patients $(28,29,31,33)$. It is possible that the implemented protocols directed clinicians away from midazolam use and hence is an expected result. The studies also showed that using less midazolam in the PICU was not harmful and resulted in higher success in meeting sedation targets (31) without an increase in adverse effects. Literature to support minimization of benzodiazepines is useful, as growing evidence suggests that benzodiazepines can have a negative impact on cognitive development and incidence of delirium $(8,10)$.

Studies have shown mixed results with regard to overall analgesic and sedative exposure. Several studies have shown an increase in sedative exposure with the implementation of protocolized sedation $(18,30,32)$, thought to be secondary to improvement in target sedative goals and reduction in under-sedation. Conversely, other studies have shown a reduction in total sedative exposure (27-29), therefore reducing over-sedation. Without knowledge of an institution's sedation practice prior to the implementation of a protocol, it is difficult to interpret the impact on cumulative sedative or analgesic dosing, as it very much reflects cultural practices within the unit; however, achieving better target sedation goals is promising.

Use of sedation protocols improves compliance with sedation assessments $(27,30,31)$ and achievement of target sedation scores within the prescribed range. An improvement in communication and documentation of sedation assessments has also been reported (20). In a survey of nurses and junior and senior physicians, all respondents reported that the introduction of a sedation protocol facilitated team decision making and resulted in more efficient and rapid intervention to ensure patient comfort (18). Another important benefit of sedation protocols is implied rather than actually measured in the studies reported. That is, the benefit of empowering the bedside clinician, generally the nurse, to act on the assessment of their patient's comfort cannot be understated.
It is generally accepted that a routine part of nursing care is to complete assessments of each patient's comfort level. Without the predefined protocol, nurses would need to first find a physician, who would then assess the patient and prescribe dose adjustments to address comfort, causing a real time delay in delivering comfort to the patient. With a sedation protocol, the bedside clinician can respond immediately to address the patient's comfort by working within predefined boundaries. This approach allows for the timely communication, documentation, and achievement of target sedation goals. This advantage is not explicitly reported in the studies but rather implied. Consideration should be given to capturing this benefit in future study designs.

\section{What about daily sedation interruption?}

Another sedation protocol that gained popularity initially in adult critical care is the concept of daily sedation interruption to minimize cumulative sedation and thereby decrease MV duration and ICU LOS (36). The results of these studies in the adult literature are mixed. Several studies showed decreases in duration of $M V$ and total cumulative dose of sedatives administered $(37,38)$; however, a larger more recent RCT showed no decreases in duration of MV or ICU LOS when protocolized sedation was combined with daily sedation interruption (39).

To date, three pediatric studies have investigated daily sedation interruption. The first two were single-center RCTs that compared daily sedation interruption to usual care. Both studies demonstrated decreased length of MV, decreased use of sedation, and shorter PICU LOS $(40,41)$. The largest study was a multicenter RCT that compared daily sedation interruption and protocol-directed sedation. The study included three tertiary hospitals in the Netherlands and 129 patients. The authors found no differences between the two groups in length of $\mathrm{MV}$, cumulative dose of benzodiazepines, or PICU LOS, and daily sedation interruption was associated with increased mortality and need for reintubation (42). Thus, unlike protocolized sedation, daily sedation interruption has not been widely adopted in the pediatric population because of the potential for adverse events.

\section{Role of sedation protocols in preventing IWS in the PICU}

It is well documented that prolonged use of analgesics and 
Table 3 PICU liberation bundle (44)

\begin{tabular}{ll}
\hline Bundle component & Description \\
\hline A & Assess, prevent, and manage pain \\
B & Spontaneous awakening trials and spontaneous breathing trials \\
C & Choice of analgesia and sedation \\
D & Delirium: assess, prevent, and manage \\
E & Early mobility and exercise \\
F & Family engagement and empowerment \\
\hline
\end{tabular}

PICU, pediatric intensive care unit.

sedatives can lead to tolerance and dependence and hence IWS if those medications are discontinued abruptly (11). Establishing a sedation protocol could potentially prevent IWS by reducing the total cumulative doses of medications or by building an active weaning process into the protocol as guided by sedation targets. Validated withdrawal scores are available for use in pediatrics. These include the Withdrawal Assessment Tool-1 (WAT-1) and the Sophia Observational Withdrawal Symptoms scale (11). In one study, introduction of a sedation protocol significantly reduced the incidence of IWS from $23.6 \%$ to $12.8 \%$ (29). Other studies have reported trends toward reduction of withdrawal symptoms after introduction of a sedation protocol $(21,27)$. In the RESTORE study, no difference was seen in the incidence of IWS (25). Despite mixed results, prevention of IWS is an important consideration for future studies.

\section{Role of sedation protocols within PICU liberation bundles}

As mortality in the PICU has decreased, several quality improvement initiatives have been aimed at mitigating the long-term psychologic, social, and physical impairment of critical illness (43). ICU liberation bundles, also known as ABCDEF Bundles (44) (Table 3), are evidence-based guidelines to liberate patients from the harmful effects of a PICU stay $(10,43)$. It should be noted that sedation protocols are a critical element of these bundles, directly addressing " $\mathrm{A}$ " and " $\mathrm{C}$ " and having an impact on "B", "D", and "E". Special consideration should be given to the screening and diagnosis of delirium ("D" in the liberation bundle) when utilizing sedation protocols. A number of delirium symptoms overlap with those observed with pain, distress and withdrawal, thus integration of validated delirium screening is essential. There are two validated commonly used bedside screening tools for delirium in pediatric intensive care patients: The Pediatric Confusion Assessment Method for the ICU (pCAM-ICU or psCAM-ICU for preschool-age children) and the Cornell Assessment of Pediatric Delirium (CAPD) (10,11). A review of the diagnosis and management of delirium is outside the scope of this review, but future research must assess sedation protocols as a component of multifaceted strategies to improve PICU outcomes.

\section{Barriers and sustainability of sedation protocols}

Institutional implementation of sedation protocols through quality improvement initiatives has been associated with numerous challenges. In one study, Yaghmai et al. (32) aimed to evaluate whether the initial benefits of their institution's sedation protocol persisted after a 4-year period. When the sedation protocol tool was initially introduced in 2008, implementation reduced LOS, sedation days, and number of opioid infusion days and decreased benzodiazepine use (33). However, the only benefit maintained was the reduction in benzodiazepine dose (32). The authors concluded that sustainability is an important consideration when creating a sedation protocol and that it requires clinical champions to carry it forward, a robust education program, ongoing quality assessment, and consistent audits (32). Other barriers to implementation have also been described, including lack of agreement and familiarity with protocol design $(18,30)$ and challenges with unit culture change around sedation minimization (30). Reluctance by staff to reduce sedation at night caused one institution to halt protocol use overnight (30). Barriers to implementation and sustainability of sedation protocols are difficult to study. Additionally, it is challenging to capture 
reasons behind deviations from a protocol in the traditional study designs used to date. Adherence to protocol and preservation of protocol integrity over time are important considerations for future studies.

\section{Challenges with research in sedation}

As we have described above, there are many challenges associated with conducting research to better understand sedation in critical care (36). A high degree of individual variability is exacerbated by the wide age range and differences in pharmacokinetics and pharmacodynamics in the pediatric population. The variability in intensity of nursing and medical bedside care and drugs that are available internationally also adds to the challenge of studying this topic (36). The success of a sedation minimization strategy depends on factors influenced by unit culture, including local practices, nurse-to-patient ratios, and intensity of training and experience (36), which is not always translatable or easy to study. Designing studies to address these factors can also be challenging because of the multicomponent structure of such an intervention (45), including patient monitoring and assessment, measurement and evaluation of sedation, practitioner education and training, and interprofessional collaboration (45). Finally, the most commonly used study designs have all compared sedation protocols to "usual care", a term that implies a uniform practice standard (45). In reality, though, the substantial variability and inconsistency in usual care makes any comparison challenging to study.

\section{Conclusions}

Strong evidence from available literature supports the premise that pediatric patients can safely tolerate minimal effective sedation with the use of a sedation protocol. No increases in adverse outcomes have been attributed to a decrease in sedation. Other reported benefits include improved communication, targeting of sedation goals, reduction of benzodiazepine use, and a reduction in IWS. A review of the evidence, however, does not reveal a clear benefit to protocol-directed sedation or daily sedation interruption over usual care in duration of MV, LOS, or mortality. Nevertheless, protocolized sedation is gradually becoming part of the accepted milieu of the PICU, as it can facilitate interventions that do impact PICU outcomes such as early mobility, reduction in delirium, and family interaction. Future studies should consider the incorporation of targeted sedation delivery in the context of bundled care in the assessment of PICU outcomes.

\section{Acknowledgments}

We would like to thank Claire Levine, MS for her editorial assistance with this manuscript.

Funding: None.

\section{Footnote}

Provenance and Peer Review: This article was commissioned by the Guest Editors (Jan Hau Lee, Vijay Srinivasan, and Debbie Long) for the series "Pediatric Critical Care" published in Translational Pediatrics. The article has undergone external peer review.

Conflicts of Interest: All authors have completed the ICMJE uniform disclosure form (available at http://dx.doi. org/10.21037/tp-20-328). The series "Pediatric Critical Care" was commissioned by the editorial office without any funding or sponsorship. The authors have no other conflicts of interest to declare.

Ethical Statement: The authors are accountable for all aspects of the work in ensuring that questions related to the accuracy or integrity of any part of the work are appropriately investigated and resolved.

Open Access Statement: This is an Open Access article distributed in accordance with the Creative Commons Attribution-NonCommercial-NoDerivs 4.0 International License (CC BY-NC-ND 4.0), which permits the noncommercial replication and distribution of the article with the strict proviso that no changes or edits are made and the original work is properly cited (including links to both the formal publication through the relevant DOI and the license). See: https://creativecommons.org/licenses/by-nc-nd/4.0/.

\section{References}

1. Barnes S, Yaster M, Kudchadkar SR. Pediatric sedation management. Pediatr Rev 2016;37:203-12.

2. Keogh SJ, Long DA, Horn DV. Practice guidelines for sedation and analgesia management of critically ill children: a pilot study evaluating guideline impact and feasibility in the PICU. BMJ Open 2015;5:e006428.

3. Playfor S, Jenkins I, Boyles C, et al. Consensus guidelines 
on sedation and analgesia in critically ill children. Intensive Care Med 2006;32:1125-36.

4. Lucas SS, Nasr VG, Ng AJ, et al. Pediatric Cardiac Intensive Care Society 2014 Consensus Statement: Pharmacotherapies in Cardiac Critical Care: Sedation, Analgesia and Muscle Relaxant. Pediatr Crit Care Med 2016;17:S3-15.

5. Kudchadkar SR, Yaster M, Punjabi NM. Sedation, sleep promotion, and delirium screening practices in the care of mechanically ventilated children: a wake-up call for the pediatric critical care community*. Crit Care Med 2014;42:1592-600.

6. Vet NJ, Ista E, de Wildt SN, et al. Optimal sedation in pediatric intensive care patients: a systematic review. Intensive Care Med 2013;39:1524-34.

7. Tobias JD. Tolerance, withdrawal, and physical dependency after long-term sedation and analgesia of children in the pediatric intensive care unit. Crit Care Med 2000;28:2122-32.

8. Zuppa AF, Curley MAQ. Sedation analgesia and neuromuscular blockade in pediatric critical care: overview and current landscape. Pediatr Clin North Am 2017;64:1103-16.

9. Patel AK, Bell MJ, Traube C. Delirium in pediatric critical care. Pediatr Clin North Am 2017;64:1117-32.

10. Walker T, Kudchadkar SR. Pain and Sedation Management: 2018 Update for the Rogers' Textbook of Pediatric Intensive Care. Pediatr Crit Care Med 2019;20:54-61.

11. Harris J, Ramelet AS, van Dijk M, et al. Clinical recommendations for pain, sedation, withdrawal and delirium assessment in critically ill infants and children: an ESPNIC position statement for healthcare professionals. Intensive Care Med 2016;42:972-86.

12. Baarslag MA, Allegaert K, Knibbe CA, et al. Pharmacological sedation management in the paediatric intensive care unit. J Pharm Pharmacol 2017;69:498-513.

13. Curley MA, Harris SK, Fraser KA, et al. State Behavioral Scale: a sedation assessment instrument for infants and young children supported on mechanical ventilation. Pediatr Crit Care Med 2006;7:107-14.

14. Ambuel B, Hamlett KW, Marx CM, et al. Assessing distress in pediatric intensive care environments: the COMFORT scale. J Pediatr Psychol 1992;17:95-109.

15. Ista E, van Dijk M, Tibboel D, et al. Assessment of sedation levels in pediatric intensive care patients can be improved by using the COMFORT "behavior" scale. Pediatr Crit Care Med 2005;6:58-63.
16. Kerson AG, DeMaria R, Mauer E, et al. Validity of the Richmond Agitation-Sedation Scale (RASS) in critically ill children. J Intensive Care 2016;4:65.

17. Aitken LM, Bucknall T, Kent B, et al. Protocol-directed sedation versus non-protocol-directed sedation in mechanically ventilated intensive care adults and children. Cochrane Database Syst Rev 2018;11:CD009771.

18. Alexander E, Carnevale FA, Razack S. Evaluation of a sedation protocol for intubated critically ill children. Intensive Crit Care Nurs 2002;18:292-301.

19. Devlin JW, Skrobik Y, Gelinas C, et al. Clinical practice guidelines for the prevention and management of pain, agitation/sedation, delirium, immobility, and sleep disruption in adult patients in the ICU. Crit Care Med 2018;46:e825-73.

20. Larson GE, Arnup SJ, Clifford M, et al. How does the introduction of a pain and sedation management guideline in the paediatric intensive care impact on clinical practice? A comparison of audits pre and post guideline introduction. Aust Crit Care 2013;26:118-23. Erratum in: Aust Crit Care 2014;27:69.

21. Jin HS, Yum MS, Kim SL, et al. The efficacy of the COMFORT scale in assessing optimal sedation in critically ill children requiring mechanical ventilation. J Korean Med Sci 2007;22:693-7.

22. Mansouri P, Javadpour S, Zand F, et al. Implementation of a protocol for integrated management of pain, agitation, and delirium can improve clinical outcomes in the intensive care unit: a randomized clinical trial. J Crit Care 2013;28:918-22.

23. Bucknall TK, Manias E, Presneill JJ. A randomized trial of protocol-directed sedation management for mechanical ventilation in an Australian intensive care unit. Crit Care Med 2008;36:1444-50.

24. Brook AD, Ahrens TS, Schaiff R, et al. Effect of a nursingimplemented sedation protocol on the duration of mechanical ventilation. Crit Care Med 1999;27:2609-15.

25. Curley MA, Wypij D, Watson RS, et al. Protocolized sedation vs usual care in pediatric patients mechanically ventilated for acute respiratory failure: a randomized clinical trial. JAMA 2015;313:379-89.

26. Watson RS, Asaro LA, Hertzog JH, et al. Long-term outcomes after protocolized sedation versus usual care in ventilated pediatric patients. Am J Respir Crit Care Med 2018;197:1457-67.

27. Dreyfus L, Javouhey E, Denis A, et al. Implementation and evaluation of a paediatric nurse-driven sedation protocol in a paediatric intensive care unit. Ann Intensive 
Care 2017;7:36.

28. Gaillard-Le Roux B, Liet JM, Bourgoin P, et al. Implementation of a nurse-driven sedation protocol in a PICU decreases daily doses of midazolam. Pediatr Crit Care Med 2017;18:e9-17.

29. Neunhoeffer F, Kumpf M, Renk H, et al. Nursedriven pediatric analgesia and sedation protocol reduces withdrawal symptoms in critically ill medical pediatric patients. Paediatr Anaesth 2015;25:786-94.

30. Ista E, de Hoog M, Tibboel D, et al. Implementation of standard sedation management in paediatric intensive care: effective and feasible? J Clin Nurs 2009;18:2511-20.

31. Larson GE, McKeever S. Nurse titrated analgesia and sedation in intensive care increases the frequency of comfort assessment and reduces midazolam use in paediatric patients following cardiac surgery. Aust Crit Care 2018;31:31-6.

32. Yaghmai BF, Di Gennaro JL, Irby GA, et al. A pediatric sedation protocol for mechanically ventilated patients requires sustenance beyond implementation. Pediatr Crit Care Med 2016;17:721-6.

33. Deeter KH, King MA, Ridling D, et al. Successful implementation of a pediatric sedation protocol for mechanically ventilated patients. Crit Care Med 2011;39:683-8.

34. Poh YN, Poh PF, Buang SN, et al. Sedation guidelines, protocols, and algorithms in PICUs: a systematic review. Pediatr Crit Care Med 2014;15:885-92.

35. Pollack MM, Holubkov R, Funai T, et al. Pediatric intensive care outcomes: development of new morbidities during pediatric critical care. Pediatr Crit Care Med 2014;15:821-7.

36. Shehabi Y, Bellomo R, Mehta S, et al. Intensive care sedation: the past, present and the future. Crit Care 2013;17:322.

Cite this article as: Balit CR, LaRosa JM, Ong JSM, Kudchadkar SR. Sedation protocols in the pediatric intensive care unit: fact or fiction? Transl Pediatr 2021;10(10):2814-2824. doi: $10.21037 / \mathrm{tp}-20-328$
37. Kress JP, Pohlman AS, O'Connor MF, et al. Daily interruption of sedative infusions in critically ill patients undergoing mechanical ventilation. $\mathrm{N}$ Engl J Med 2000;342:1471-7.

38. Girard TD, Kress JP, Fuchs BD, et al. Efficacy and safety of a paired sedation and ventilator weaning protocol for mechanically ventilated patients in intensive care (Awakening and Breathing Controlled trial): a randomised controlled trial. Lancet 2008;371:126-34.

39. Mehta S, Burry L, Cook D, et al. Daily sedation interruption in mechanically ventilated critically ill patients cared for with a sedation protocol: a randomized controlled trial. JAMA 2012;308:1985-92.

40. Gupta K, Gupta VK, Jayashree M, et al. Randomized controlled trial of interrupted versus continuous sedative infusions in ventilated children. Pediatr Crit Care Med 2012;13:131-5. Erratum in: Pediatr Crit Care Med 2012;13:373.

41. Verlaat CW, Heesen GP, Vet NJ, et al. Randomized controlled trial of daily interruption of sedatives in critically ill children. Paediatr Anaesth 2014;24:151-6.

42. Vet NJ, de Wildt SN, Verlaat CW, et al. A randomized controlled trial of daily sedation interruption in critically ill children. Intensive Care Med 2016;42:233-44.

43. Walz A, Canter MO, Betters K. The ICU Liberation Bundle and Strategies for Implementation in Pediatrics. Curr Pediatr Rep 2020. [Epub ahead of print]. doi: 10.1007/s40124-020-00216-7.

44. Society of Critical Care Medicine. ICU Liberation (Internet). USA. (cited 2021 Mar 22). Available online: https://www.sccm.org/ICULiberation/ABCDEF-Bundles

45. Blackwood B, Tume L. The implausibility of 'usual care' in an open system: sedation and weaning practices in Paediatric Intensive Care Units (PICUs) in the United Kingdom (UK). Trials 2015;16:325. 